\title{
Optimization with PDE Constraints
}

\section{Hinze}

Universität Hamburg

Germany

R. Pinnau

Technische Universität Kaiserslautern

Germany

\section{Ulbrich}

Technische Universität München

Germany

and

S. Ulbrich

Technische Universität Darmstadt

Germany

\section{算 Springer}




\section{Contents}

Preface $\ldots \ldots \ldots \ldots \ldots \ldots \ldots \ldots \ldots$

1 Analytical Background and Optimality Theory . . . . . . . . . 1

Stefan Ulbrich . . . . . . . . . . . . . . . . . . . . . . . 1

1.1 Introduction and Examples $\ldots \ldots \ldots \ldots \ldots$

1.1.1 Introduction . . . . . . . . . . . . 1

1.1.2 Examples for Optimization Problems with PDEs . . . . . 4

1.1.3 Optimization of a Stationary Heating Process . . . . . . 5

1.1.4 Optimization of an Unsteady Heating Processes . . . . . 7

1.1.5 Optimal Design . . . . . . . . . . . . . . . . 8

1.2 Linear Functional Analysis and Sobolev Spaces . . . . . . . . 9

1.2.1 Banach and Hilbert Spaces . . . . . . . . . . . . 10

1.2.2 Sobolev Spaces . . . . . . . . . . . . . . . . . . . 13

1.2.3 Weak Convergence ... . . . . . . . . . . . . 24

1.3 Weak Solutions of Elliptic and Parabolic PDEs . . . . . . . . 26

1.3.1 Weak Solutions of Elliptic PDEs . . . . . . . . 26

1.3.2 Weak Solutions of Parabolic PDEs . . . . . . . . . 36

1.4 Gâteaux- and Fréchet Differentiability . . . . . . . . . 50

1.4.1 Basic Definitions . . . . . . . . . . . . . . . . 50

1.4.2 Implicit Function Theorem . . . . . . . . . . . 52

1.5 Existence of Optimal Controls . . . . . . . . . . . . . . . 52

1.5.1 Existence Result for a General Linear-Quadratic

Problem . . . . . . . . . . . . . . 52

1.5.2 Existence Results for Nonlinear Problems . . . . . . . . 54

1.5.3 Applications . . . . . . . . . . . . . . . . 56

1.6 Reduced Problem, Sensitivities and Adjoints . . . . . . . . 57

1.6.1 Sensitivity Approach . . . . . . . . . . . . . . . 58

1.6.2 Adjoint Approach . . . . . . . . . . . . . . . . . 59

1.6.3 Application to a Linear-Quadratic Optimal Control

Problem . . . . . . . . . . . . . . . 60

1.6.4 A Lagrangian-Based View of the Adjoint Approach . . . . 63 
1.6.5 Second Derivatives . . . . . . . . . . . . . . . 64

1.7 Optimality Conditions . . . . . . . . . . . . . . 65

1.7.1 Optimality Conditions for Simply Constrained Problems . . 65

1.7.2 Optimality Conditions for Control-Constrained Problems . . . . . . . . . . . . . . . . . . 70

1.7.3 Optimality Conditions for Problems with General

Constraints . . . . . . . . . . . . . . . 80

1.8 Optimal Control of Instationary Incompressible Navier-Stokes

Flow . . . . . . . . . . . . . . . . . 88

1.8.1 Functional Analytic Setting . . . . . . . . . . . . 89

1.8.2 Analysis of the Flow Control Problem . . . . . . . . 91

1.8.3 Reduced Optimal Control Problem . . . . . . . . . . . . 94

2 Optimization Methods in Banach Spaces . . . . . . . . . . . 97

Michael Ulbrich . . . . . . . . . . . . . . . . . . 97

2.1 Synopsis . . . . . . . . . . . . . . . . . . . 97

2.2 Globally Convergent Methods in Banach Spaces . . . . . . . . . 99

2.2.1 Unconstrained Optimization . . . . . . . . . . . . . . . . . 99

2.2.2 Optimization on Closed Convex Sets . . . . . . . . . . 104

2.2.3 General Optimization Problems . . . . . . . . . . . . . 109

2.3 Newton-Based Methods-A Preview . . . . . . . . . . . . . 109

2.3.1 Unconstrained Problems - Newton's Method . . . . . . . . 109

2.3.2 Simple Constraints . . . . . . . . . . . . . . . . 110

2.3.3 General Inequality Constraints . . . . . . . . . . 113

2.4 Generalized Newton Methods . . . . . . . . . . . . . . . . 115

2.4.1 Motivation: Application to Optimal Control . . . . . . . 115

2.4.2 A General Superlinear Convergence Result . . . . . . . . 116

2.4.3 The Classical Newton's Method . . . . . . . . . . . . . . 119

2.4.4 Generalized Differential and Semismoothness . . . . . . 120

2.4.5 Semismooth Newton Methods . . . . . . . . . . . . . . 123

2.5 Semismooth Newton Methods in Function Spaces . . . . . . . 125

2.5.1 Pointwise Bound Constraints in $L^{2} \ldots \ldots \ldots \ldots$. . . 125

2.5.2 Semismoothness of Superposition Operators . . . . . . . 126

2.5.3 Pointwise Bound Constraints in $L^{2}$ Revisited . . . . . . . . 129

2.5.4 Application to Optimal Control . . . . . . . . . . . . . 130

2.5.5 General Optimization Problems with Inequality Constraints in $L^{2} \ldots \ldots \ldots \ldots$. . . . . . . . 132

2.5.6 Application to Elliptic Optimal Control Problems . . . . 133

2.5.7 Optimal Control of the Incompressible Navier-Stokes

Equations . . . . . . . . . . . . . . . 137

2.6 Sequential Quadratic Programming . . . . . . . . . . . . . . . 140

2.6.1 Lagrange-Newton Methods for Equality Constrained

Problems . . . . . . . . . . . . . . . . . . . 140

2.6.2 The Josephy-Newton Method . . . . . . . . . . . . . . . . 144

2.6.3 SQP Methods for Inequality Constrained Problems . . . . . 148 
2.7 State-Constrained Problems . . . . . . . . . . . . . . . 151

2.7 .1 SQP Methods . . . . . . . . . . . . . . . . 152

2.7.2 Semismooth Newton Methods . . . . . . . . . . . . . 152

2.8 Further Aspects . . . . . . . . . . . . . . . . 155

2.8 .1 Mesh Independence . . . . . . . . . . . . . . . 155

2.8.2 Application of Fast Solvers . . . . . . . . . . 156

2.8 .3 Other Methods . . . . . . . . . . . . . . 156

3 Discrete Concepts in PDE Constrained Optimization . . . . . . . 157

Michael Hinze . . . . . . . . . . . . . . . . . . . 157

3.1 Introduction . . . . . . . . . . . . . . . 157

3.2 Control Constraints . . . . . . . . . . . . . 158

3.2.1 Stationary Model Problem . . . . . . . . . . . . . . 158

3.2.2 First Discretize, Then Optimize . . . . . . . . . . . 160

3.2.3 First Optimize, Then Discretize . . . . . . . . . . . . 161

3.2.4 Discussion and Implications . . . . . . . . . . . . . . 163

3.2.5 The Variational Discretization Concept . . . . . . . . . . 164

3.2 .6 Error Estimates . . . . . . . . . . . . . . . . . . . 167

3.2.7 Boundary Control . . . . . . . . . . . . . . . . . 177

3.2.8 Some Literature Related to Control Constraints . . . . . . . 196

3.3 Constraints on the State . . . . . . . . . . . . . . 197

3.3.1 Pointwise Bounds on the State . . . . . . . . . . 198

3.3.2 Pointwise Bounds on the Gradient of the State . . . . . . . 219

3.4 Time Dependent Problem . . . . . . . . . . . . . . . . 227

3.4.1 Mathematical Model, State Equation . . . . . . . . . 227

3.4 .2 Optimization Problem . . . . . . . . . . . . . . . . . . . . . . . . . . . . . . . . . .

3.4 .3 Discretization . . . . . . . . . . . . . . . . . . 229

3.4.4 Further Literature on Control of Time-Dependent

Problems . . . . . . . . . . . . . . . . 231

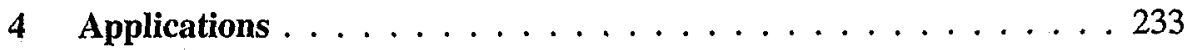

René Pinnau . . . . . . . . . . . . . . . . . . 233

4.1 Optimal Semiconductor Design . . . . . . . . . . . . 233

4.1.1 Semiconductor Device Physics . . . . . . . . . . . 234

4.1.2 The Optimization Problem . . . . . . . . . . . . 240

4.1 .3 Numerical Results . . . . . . . . . . . . . . . . . . . 246

4.2 Optimal Control of Glass Cooling . . . . . . . . . . . 250

4.2 .1 Modeling . . . . . . . . . . . . . . 251

4.2.2 Optimal Boundary Control . . . . . . . . . . . 254

4.2 .3 Numerical Results . . . . . . . . . . . . . . . 260



